\title{
An Updated CLAHE Approach for Image Enhancement with Advanced Wavelets
}

\author{
Tarandeep $^{1}$, Er. Varinderjit Kaur ${ }^{2}$, Dr. Naveen Dhillon ${ }^{3}$ \\ Dept of Computer Science and Engg, Ramgarhia Institute of Engg and Tech, Phagwara, Punjab ${ }^{1}$ \\ HOD, Dept of Computer Science and Engg, Ramgarhia Institute of Engg and Tech, Phagwara, Punjab ${ }^{2}$ \\ Principal, RIET Ramgarhia Institute of Engg and Tech, Phagwara, Punjab ${ }^{3}$
}

\begin{abstract}
Image enhancement is a process which performs some operation on the image in order to obtain high contrast images with better quality by suppressing the noisy pixels. Quality of the image is a satisfactory part of any image enhancement technique. Consequently, several techniques till now have been proposing to acquire high quality image while performing different operations. Traditional techniques are not able to preserve the edges of the image which degrades the quality of the image and no longer able to use for other purposes. Owing to this a new technique has proposed which makes quality oriented image after enhancing contrast. A hybrid technique has applied on the image which considers every part of the image and preserves edges too. The proposed method provides good result in terms of different images i.e. gray scale or RGB color images as compared to traditional techniques. Experiments have performed to estimate the performance of the proposed technique visually.
\end{abstract}

Keywords: Image enhancement, CLAHE, SWT, color space.

\section{INTRODUCTION}

Image enhancement or digital image processing plays an important role for increasing the quality and other features of the image. Image processing includes different processes such as image enhancement, image analysis, image denoising, detection; image segmentation etc. Among that Image enhancement is done for increasing the visual aspect of an image. There is a need of image preprocessing in some cases where image poses the low dynamic range of the intensity value due to lack of elucidation. There are many techniques for image enhancements have been proposed and most of them focus on enhancing the grey level images using spatial domain. Example of these techniques is histogram equalization; low pass and high pass filtering techniques etc. Contrast enhancement is the process of image enhancement which brings attention of many researchers. Contrast enhancement increases the quality of the image from visual point of view. It has wide variety of applications such as speech recognition, medical image preprocessing and other fields of image or video preprocessing. Mainly used methods or techniques for contrast enhancement is HE i.e. Histogram Equalization because of its efficient distribution of pixel intensity with respect to full intensity range. The main advantage of this technique is that it consists less complex calculations and easy to recover such as if the histogram equalization function is notorious then it would become easy to observe the original histogram. The disadvantage of this technique is that it has an inequality problem which leads to the enhancement in the contrast of noise at the time of reducing usable signals. Image enhancement is divided into two parts on the basis of domain. These are as follows:
1. Spatial Domain.

2. Frequency Domain.

Spatial Domain applies the alterations directly on the image plane i.e. it applies modifications on the pixel values directly. It is also known as direct manipulation on the image surface.

Frequency Domain is used for making changes in the Fourier transform of an image. Spatial domain works on the basis of aggregation technique

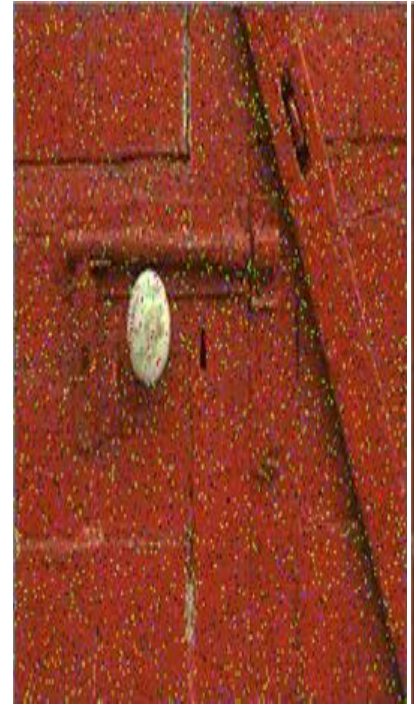

(a)

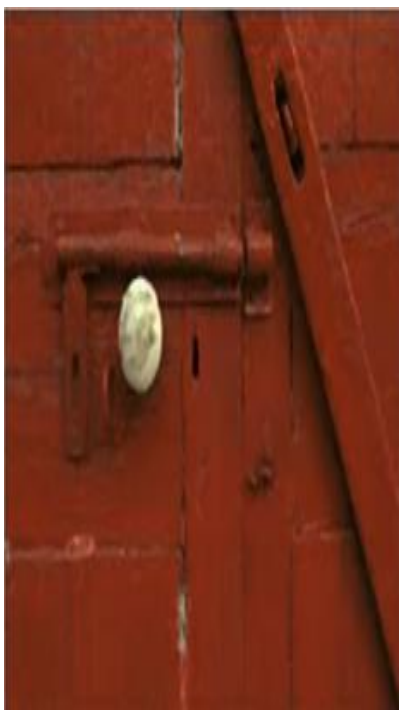

(b)
Figure 1.Image Enhancement (a) Noisy image (b) Enhanced Image 
Vol. 6, Issue 1, January 2017

This paper has proposed a classification of HE technique along with SWT technique to improve the visual quality of the image by suppressing the noise from the acquired image at the time of acquisition.

\section{PROBLEM STATEMENT}

Image enhancement is the technique of enhancing the quality of image by improving the quality of its pixels. Image enhancement is a method helpful in improving the quality of the image by improving contrast and brightness with reduction in noise content or sharpens its detail. The objective of applying image enhancement technique is to make the image suitable for a specific task and observer by modifying the attributes of the image. Methods that were developed for image enhancement were spatial domain methods and frequency domain methods. In these spatial domain methods the image pixels were directly used for enhancing the quality of image whereas in frequency domain methods, the image is first converted to the frequency domain. Traditionally the DWT technique was used for the image enhancement. The major drawback of using DWT was that the edges of the image were not preserved that decrease the quality of the image. So there is need of proposing new method that is better and efficient than the existing method of image enhancement. An efficient technique can be designed using the ideology of the conventionally used techniques and can help in enhancing the image to high extent.

The main objectives of the proposed work are:

1. To study existing techniques for image enhancement and evaluate the problems in the existing techniques.

2. To implement technique of image enhancement based on CLAHE-SWT in order to use benefits of both existing techniques and proposed a new technique.

3. To design a new technique with more efficiency in image enhancement using Lab color space.

4. Performance Analysis of the proposed technique.

\section{PROPOSED WORK}

The main aim of using the technique for image enhancement is enhancing the quality of image to obtain a better quality image from the available image. Numbers of techniques are used conventionally for image enhancement but the drawback of these conventional techniques was that the edges of the image were not preserved A technique needs to be developed that will enhance the available image and will produce a high quality image after enhancement.

A hybrid approach is produced that is developed using two conventional techniques that are SWT and the CLAHE. The CLAHE protocol is applied on the image in which LAB space color conversion is done. The proposed technique enhances the image and it helps in achieving high quality image after enhancement.

\section{- CLAHE}

CLAHE considered as Contrast limited adaptive histogram equalization which provides clip limit so to overcome the problem of noise in the image. CLAHE is performed by dividing block size and clip limit. These defined parameters have been used to control the quality of the image. CLAHE does not perform on the whole image so it performs on small regions of the image also considered as tiles. Thus enhancement has been performed on the small regions. After performing histogram it has been combined by using bilinear interpolation. This process will be helpful in abolishing artificially induced boundaries. CLAHE was used in medical imaging and to enhance the low contrast images such as portal films. Equation used for standard CLAHE method with uniform Distribution expressed as:

$$
\mathrm{g}=\left[\mathrm{g}_{\max }-\mathrm{g}_{\min }\right] * \mathrm{P}(\mathrm{f})+\mathrm{g}_{\min }
$$

where $g_{\max }=$ maximum pixel value

$\mathrm{g}_{\min }=$ Minimum pixel value

$\mathrm{g}=$ computed pixel value

$\mathrm{P}(\mathrm{f})=\mathrm{CPD}$ (Cumulative Probability Distribution)

For exponential distribution gray level can be adapted as:

$$
\mathrm{g}=\mathrm{g}_{\min }-\left(\frac{1}{\alpha}\right) * \operatorname{In}[1-(\mathrm{f})]
$$

Where, $\alpha$ is the clip parameter

\section{- SWT}

Stationary Wavelet Transform is the technique used for enhancement of the image. This technique is based on the Discrete Wavelet Transform rather than Time variant transform. To define the SWT, un-decimated DWT can be used. Suppression has been performed through down sampling step of the decimated algorithm rather than using up down sampling filters. Following is the algorithm used to implement SWT:

1. Convert the original image into subbands i.e. three detail subbands and one approximation sub band such as HL, LH, HH and LL bands using SWT at one level.

2. Then average of the approximate parts of the image will be taken.

3. Absolute values of the horizontal details of the image will be taken and second part will be subtracted from the first one using

$$
\mathrm{D}=(\operatorname{abs}(\mathrm{H} 1 \mathrm{~L} 2)-\mathrm{abs}(\mathrm{H} 2 \mathrm{~L} 2))>=0
$$

4. In order to obtain fused horizontal part of the image, make element wise multiplication of $\mathrm{D}$ and then take horizontal detail of the first image then do subtract it with another horizontal detail of the second image. Thus second image is multiplied by logical not of D from first.

5. Obtain fused vertical and details of the images. Found $\mathrm{D}$ for vertical and diagonal parts.

6. Whole steps will be repeated for fusion at first level.

7. Lastly, perform Inverse Stationary Wavelet Transform. 


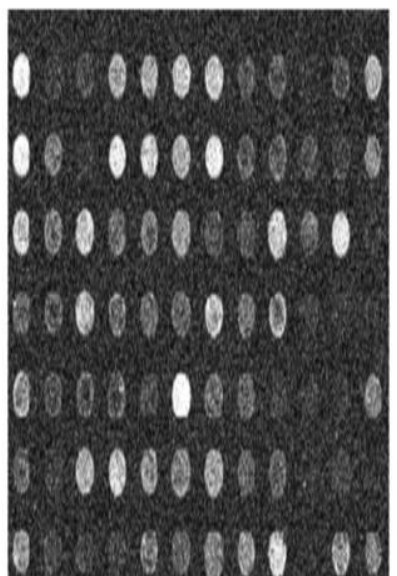

(a)

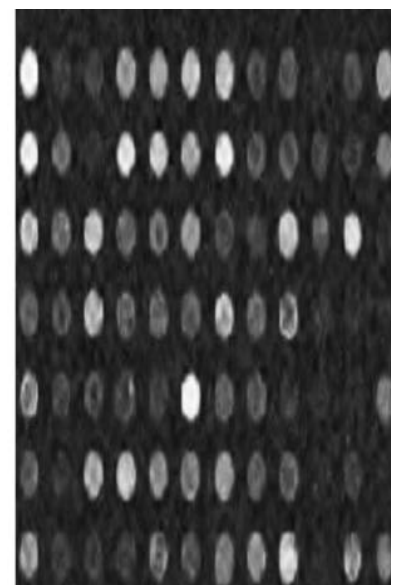

(b)
Figure2. Example of image enhancement using SWT (a) original image (b) Denoised image after applying SWT

In the proposed technique CLAHE will be applied in order to enhance the contrast and intensity of the image and SWT will be used to separate the layers of the image such as Approximation, Horizontal, Vertical and Diagonal. So combination of these two techniques perform enhancement.

\section{METHODOLOGY}

In the proposed work, contrast of the image is enhanced using enhancement technique. Hybrid technique has been applied for better picture quality. The methodology of the proposed technique is mentioned below:

1. Initially browse an image from the database to perform enhancement. An image will be loaded into the MATLAB for further processing.

2. Now apply SWT i.e. Stationary Wavelet Transform on the image taken from the database.

3. After applying SWT, three layers of the images will be obtained named as A, H, V and D i.e. Approximation, Horizontal, Vertical, Diagonal respectively.

4. Now perform CLAHE Enhancement on the approximation layer of the SWT.

5. Thus obtain image from $\mathrm{A}, \mathrm{H}, \mathrm{V}$ and $\mathrm{D}$ will be fused using Inverse SWT.

6. Acquired image from the ISWT will be converted into color space known as LAB color space where LAB referred as Lightness for $\mathrm{L}$ and $\mathrm{a}$, bare color dimensions.

7. Now on the $\mathrm{L}$ layer of the image; CLAHE enhancement will be performed whereas lightness or intensity will be increased so that image obtained looks enhanced with better quality.

8. Then again convert the LAB color space format into RGB color format. As input image was in RGB format so then output image should be in the same format thus format will be converted into original format.

Lastly evaluate performance of the proposed technique by calculate parameters values. And hence shows the accuracy of the proposed technique in comparison with the existing techniques.

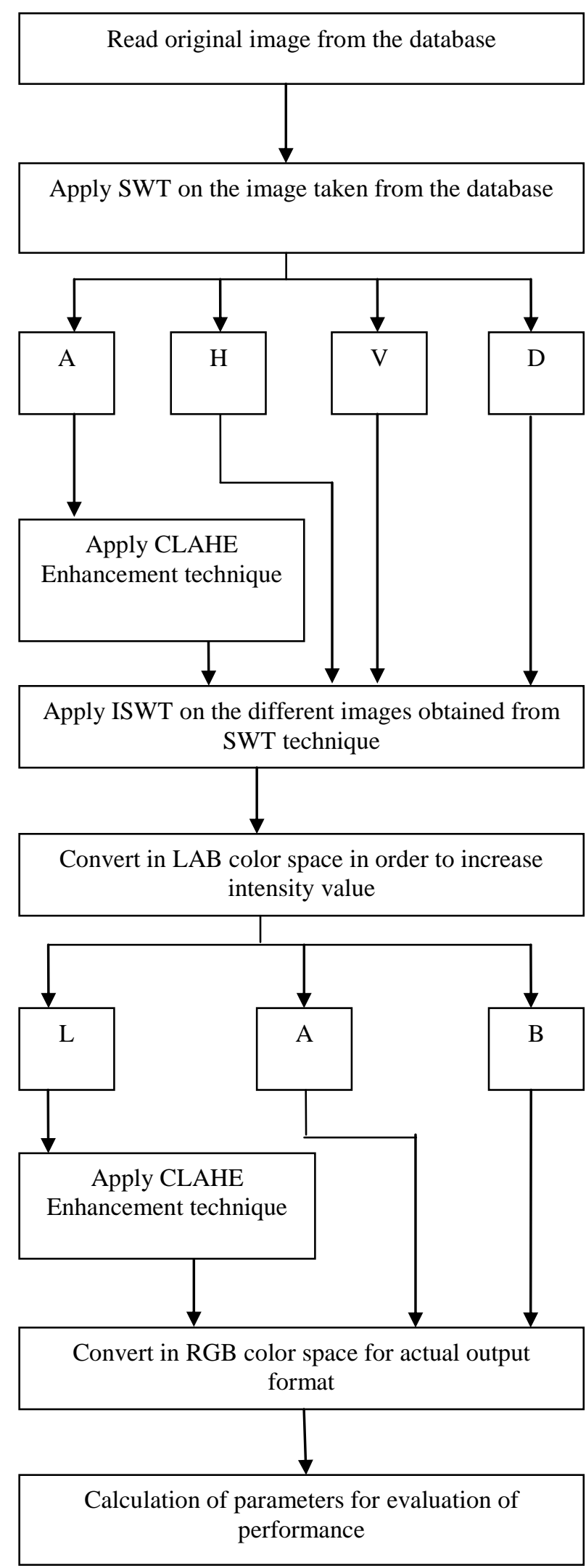

Figure 3. Flow diagram of proposed work 
Vol. 6, Issue 1, January 2017

\section{RESULTS AND DISCUSSION}

In order to prove the efficiency of proposed technique over traditional technique, the results are discussed. This section represents the results of various performance parameters. The simulation is performed in MATLAB and the technique is applied on image and compared with traditional technique.

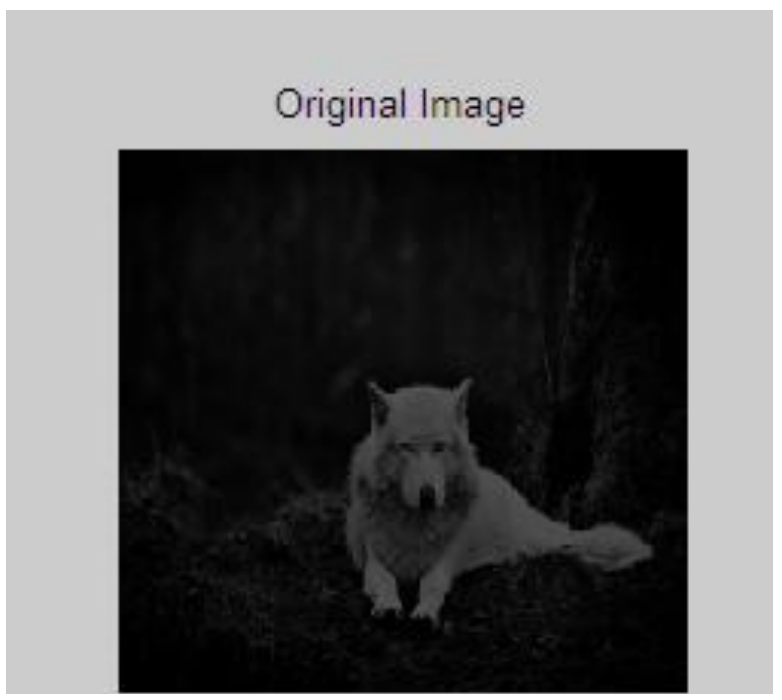

Figure 4 Original image

Different parameters like NE (noise estimation), PSNR (Peak Signal to Noise Ratio), LEI (local Entropy) and Mean Square Error i.e. MSE have been evaluated to check the performance of different techniques with respect to proposed technique.

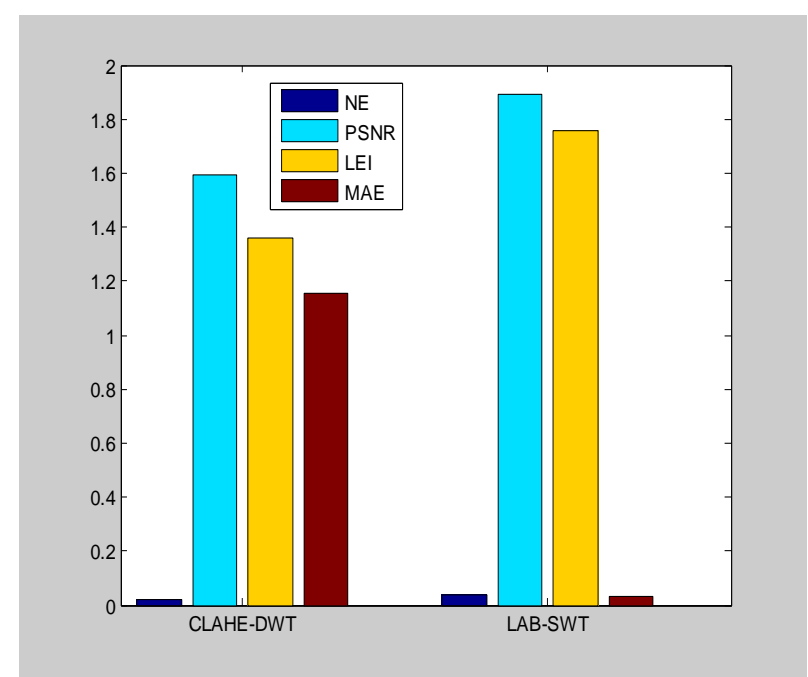

Figure 5 Represents comparison of hybridization technique with traditional one.

Parameters have applied on the original image shown in figure 5 and then results acquired for different techniques have shown in the below figure. From the figure 6 it clearly depicts that proposed technique of LAB-SWT outperforms.

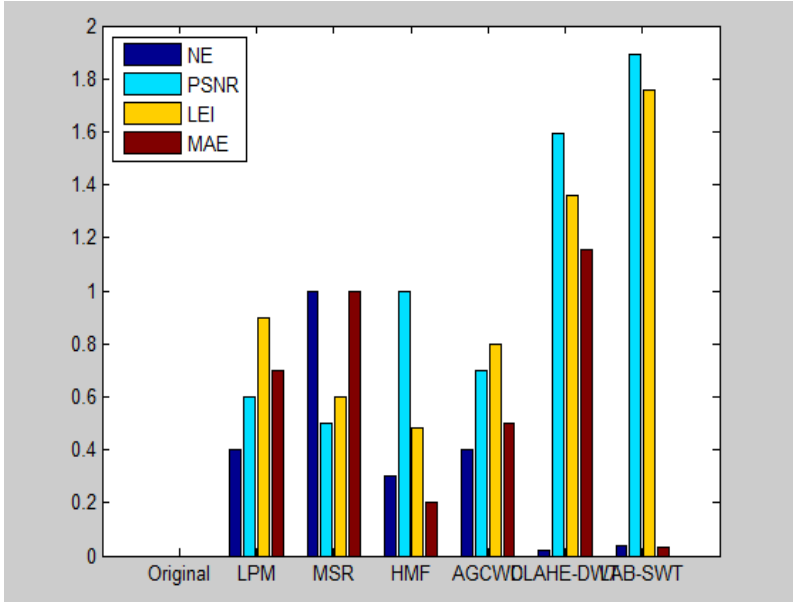

Figure6 shows performance parameter of different techniques

After performing several parameters obtained enhanced image has shown in the below figure.

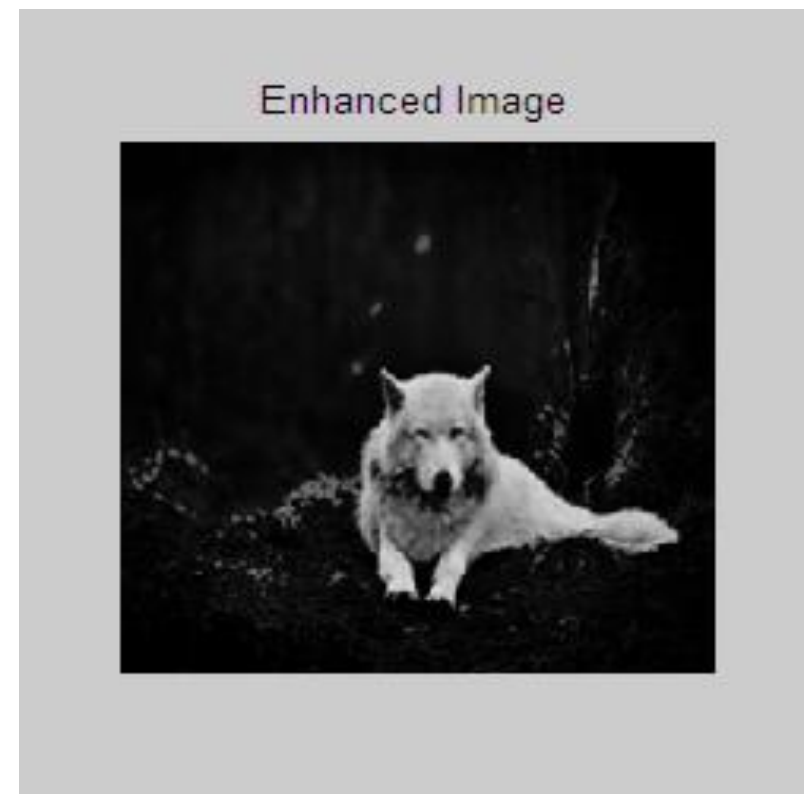

Figure 7 Enhanced image after performing manipulation.

\section{CONCLUSION AND FUTURE SCOPE}

Image enhancement is a technique which provides better visual image by suppressing noise. Initially enhancement has been applied on the Approximation layer of the image and afterward on the intensity of the image.

In this paper, hybridization technique is applied on the image in which contrast enhancement CLAHE and SWT technique has been used. SWT technique has applied due to its advantage as it transforms the image at each point and saves detail coefficients. Moreover, it uses low frequency at each level. Due to which combination of these two techniques are used. Evaluations have been performed that has proven the fact given in the proposed work. Resultant parameters show that proposed technique is better in terms of each parameter. 
Vol. 6, Issue 1, January 2017

In future, more techniques can been hanced in order to acquire high quality images so that these images can be used for several applications such as medical imaging, gene expression etc. Contrast and brightness of an image can also be improved with less number of complications.

\section{REFERENCES}

[1] Ji-Hee Han (2011), “A novel 3-d color histogram equalization method with uniform 1-d gray scale histogram "IEEE transactions on image processing, vol. 20, NO. 2, pp 506-512

[2] Omprakash Patel (2013), "A comparative study of histogram equalization based image enhancement techniques for brightness preservation and contrast enhancement", Signal \& Image Processing : An International Journal (SIPIJ) Vol.4, No.5,

[3] P.Shanmugavadivu (Dec 2011), "Modified histogram equalization for image contrast enhancement using particle swarm optimization "International Journal of Computer Science, Engineering and Information Technology (IJCSEIT), Vol.1, No.5

[4] Chahat Chaudhary, (2013)"Review of image enhancement techniques using histogram equalization " International Journal of Application or Innovation in Engineering \& Management (IJAIEM) Volume 2, Issue 5, Page 343 -349

[5] Dinesh Sonker (Aug 2013), "Comparison of Histogram Equalization Techniques for Image Enhancement of Grayscale images of Dawn and Dusk", International Journal of Modern Engineering Research (IJMER) Vol. 3, Issue. 4,pp-2476-2480

[6] Ravindra Pal Singh, (2015) "Histogram Equalization: A Strong Technique for Image Enhancement "International Journal of Signal Processing, Image Processing and Pattern Recognition Vol.8, No.8

[7] Raju. A , (2013), "Comparative Analysis of Histogram Equalization based Techniques for Contrast Enhancement and Brightness Preserving “ International Journal of Signal Processing, Image Processing and Pattern Recognition Vol.6, No. 5

[8] Mukesh C. Motwani et al (2004), "Survey of Image Denoising Techniques", In Proceedings of GSPX, pp. 27-30.

[9] Di Guo et al (2014), "Salt and Pepper Noise Removal with Noise Detection and a Patch-Based Sparse Representation", Advances in Multimedia, Vol. 2014, Pages 14.

[10] Hadi Sadoghi Yazdi et al (Sep 2010), "Impulsive Noise Suppression of Images Using Adaptive Median Filter", IJSP, Vol. 3, No. 3, Pp. 1-12.

[11] Khryashche v. et al (July 2011), "Vector Median Filter with Directional Detector for Color Image Denoising", Proceedings of the World Congress on Engineering, Vol. 2.

[12] Amarjit Roy et al (May 2014), "An improved vector median filter for impulse noise removal from color images", IJEEDC, Vol. 2, No. 5, Pp. 28-31.

[13] Sin Hoong Teoh et al (Dec 2012), "Median Filtering Frameworks for Reducing Impulse Noise from Grayscale Digital Images: A Literature Survey", IJFCC, Vol. 1, No. 4, Pp. 323-326.

[14] B. Smolka et al (2001), "Fast Modied Vector Median Filter", Pp. 570-580.

[15] M. Emre Celebi et al (2007), "A fast switching filter for impulsive noise removal from color images", JIST, Vol. 51, No. 2, Pp. 155165.

[16] Abhishek Arvind Gulhane et al (March 2012), "Noise Reduction of an Image by using Function Approximation Techniques.”, IJSCE, Vol. 2, No. 1, Pp. 60- 62 .

[17] Anisha $\mathrm{K}$ et al (Nov 2011), "Impulse noise removal from medical images using fuzzy genetic algorithm", IJMA, Vol. 3, No. 4, Pp. 93.

[18] M. Emre Celebi et al (Sep 2007), "Nonlinear vector filtering for impulsive noise removal from color images", Journal of Elect4ronic Imaging, Vol. 16, No. 3.

[19] Nirmala P. et al (2015), "Optimization of Image Denoising Filters Using Harmony Search - An Experimental Analysis”, MEJSR, Pp. 305-308.

[20] Garima Goyal et al (Jan 2013)," Review Paper on Various Filtering Techniques and Future Scope to Apply These on TEM Images", IJSRP, Vol. 3, No. 1, Pp. 1-11.
[21] E. Dinet (Dec 2007), "Color Median Filtering: a Spatially Adaptive Filter", Proceedings of Image and Vision Computing New Zealand, Pp. 71-76.

[22] Krystyna Malik et al (2012), "Improved bilateral filtering scheme for noise removal in color images", The Society of Digital Information and Wireless Communication, Pp. 118-130.

[23] G. Phani Deepti (2009) "Impulse Noise Removal from Color Images with Hopfield Neural Network and Improved Vector Median Filter".

[24] Priyanka Kamboj (2013), "A brief study of various noise model and filtering techniques", Journal of Global Research in Computer Science, Vol. 4, No. 4.

[25] Andreas Koschan et al (Oct 2001), "A Comparison of Median Filter Techniques For Noise Removal in Color Images", Proc. 7th German Workshop on Color Image Processing, Vol. 34, No. 15, Pp. 69-79. 